\title{
Fast gray matter acquisition T1 inversion recovery MRI to delineate the mammillothalamic tract for preoperative direct targeting of the anterior nucleus of the thalamus for deep brain stimulation in epilepsy
}

\author{
*Sanjeet S. Grewal, MD, ${ }^{1}$ Erik H. Middlebrooks, MD, ${ }^{2}$ Timothy J. Kaufmann, MD, ${ }^{3}$ \\ Matthew Stead, MD, PhD, ${ }^{4}$ Brian N. Lundstrom, MD, PhD, ${ }^{4}$ Gregory A. Worrell, MD, PhD, ${ }^{4}$ \\ Chen Lin, PhD, ${ }^{2}$ Serhat Baydin, MD, ${ }^{5}$ and Jamie J. Van Gompel, MD ${ }^{6}$ \\ Departments of ${ }^{1}$ Neurosurgery and ${ }^{2}$ Radiology, Mayo Clinic, Jacksonville, Florida; Departments of ${ }^{3}$ Radiology, ${ }^{4}$ Neurology, and \\ ${ }^{6}$ Neurosurgery, Mayo Clinic, Rochester, Minnesota; and ${ }^{5}$ Department of Neurosurgery, Kanuni Sultan Suleyman Research and \\ Training Hospital, Istanbul, Turkey
}

\begin{abstract}
When medically intractable epilepsy is multifocal or focal but poorly localized, neuromodulation can be useful therapy. One such technique is deep brain stimulation (DBS) targeting the anterior nucleus of the thalamus (ANT). Unfortunately, the ANT is difficult to visualize in standard MRI sequences and its indirect targeting is difficult because of thalamic variability and atrophy in patients with epilepsy. The following study describes the novel use of the fast gray matter acquisition T1 inversion recovery (FGATIR) MRI sequence to delineate the mammillothalamic tract for direct targeting of the ANT through visualizing the termination of the mammillothalamic tract in the ANT.

The day prior to surgery in a 19-year-old, right-handed woman with a 5-year history of epilepsy, MRI was performed on a 3-T Siemens Prisma scanner (Siemens AG, Healthcare Sector) using a 64-channel head and neck coil. As part of the imaging protocol, noncontrast magnetization-prepared rapid gradient echo (MP-RAGE) and diffusion tensor imaging (DTI) sequences were obtained for targeting purposes. The ANT was directly targeted using the FGATIR sequence, and bilateral Medtronic 3389 leads were placed. At the last follow-up (2 months), the patient reported an approximate $75 \%$ decrease in seizure frequency, as well as a decrease in seizure severity.
\end{abstract}

https://thejns.org/doi/abs/10.3171/2018.4.FOCUS18147

KEYWORDS DBS; epilepsy; FGATIR; anterior nucleus

$\mathrm{F}$ or patients with medically refractory focal epilepsy, surgical interventions include resection, ablation, or neuromodulation. The latter can be performed through vagus nerve stimulation, ${ }^{31}$ responsive neurostimulation, ${ }^{3}$ chronic subthreshold cortical stimulation, ${ }^{16,22}$ and deep brain stimulation (DBS). ${ }^{21}$ While many sites have been tested for DBS, including the centromedian nucleus of the thalamus (CMT), ${ }^{21}$ the most rigorously studied site is the anterior nucleus of the thalamus (ANT). ${ }^{7}$
Targeting the ANT has traditionally been accomplished using stereotactic coordinates. ${ }^{10}$ Such indirect targeting is limited because of individual variations in anatomy and is particularly challenging in epilepsy as the thalamus is known to atrophy in the setting of chronic epilepsy. $2,4,13,25,29,34$ The alternative method of direct image-based targeting suffers from the lack of an easily visualized target in the ANT. $5,14,20,28$

Herein, we present the first report on using fast gray

ABBREVIATIONS ANT = anterior nucleus of the thalamus; $C M T=$ centromedian nucleus of the thalamus; $\mathrm{CMTpf}=\mathrm{CMT} /$ parafascicular complex; DBS = deep brain stimulation; DISTAL = DBS Intrinsic Template Atlas; DTI = diffusion tensor imaging; EEG = electroencephalography; EPI = echo planar imaging; FGATIR = fast gray matter acquisition T1 inversion recovery; GRAPPA = generalized autocalibrating partial parallel acquisition; MNI = Montreal Neurological Institute; MP-RAGE = magnetization-prepared rapid gradient echo; SNR = signal-to-noise ratio; STIR = short-tau inversion recovery; VTA = volume of tissue activated.

SUBMITTED March 22, 2018. ACCEPTED April 25, 2018

INCLUDE WHEN CITING DOI: 10.3171/2018.4.FOCUS18147.

*S.S.G. and E.H.M. share first authorship. 
matter acquisition T1 inversion recovery (FGATIR) MRI to delineate the mammillothalamic tract as it terminates in the ANT and thus directly targeting the ANT.

\section{Case Report}

History and Examination

A 19-year-old, right-handed woman, who had no major risk factors for epilepsy, presented with a 5-year history of the disorder. She had a history of three different seizure types. The first type consisted of behavioral arrest, expressive aphasia, and hand automatisms characterized by shuffling of both hands. The second type of seizure consisted of a very lethargic feeling upon waking in the morning with the bed in disarray. The third type of seizure was a generalized tonic-clonic seizure. She had previously tried levetiracetam, lacosamide, topiramate, clobazam, and lamotrigine with no significant improvements and significant side effects.

Prior workup included diagnostic MRI utilizing a dedicated epilepsy protocol at $3 \mathrm{~T}$. In the left frontal periventricular white matter, a small nodule that followed gray matter signal on all sequences was identified and was consistent with periventricular nodular heterotopia (Fig. 1). She was admitted to an epilepsy monitoring unit for further evaluation. During her admission, scalp electroencephalography (EEG) revealed broadly distributed left hemispheric spike-wave discharges as well as more focal independent interictal discharges from the left frontal, temporal, and parietal head regions. Generalized $2-\mathrm{Hz}$ spike-wave discharges, often preceded by left hemispheric spike-wave discharges, were seen, as were rare bursts of paroxysmal fast activity. Her seizures were not clearly localized or lateralized, although seizure activity was believed to be more prominent over the left hemisphere. At an outside institution, the patient had undergone magnetoencephalography with simultaneous EEG recording showing frequent multifocal interictal epileptiform discharges involving the left frontal, temporal, and central parietal head regions as well as the right temporal lobe. This study also showed left hemispheric language dominance.

In an attempt to further localize seizure origin, the patient underwent stereo EEG monitoring. There were frequent interictal discharges that occurred synchronously involving the left hippocampus, left middle temporal gyrus, left middle frontal gyrus, left supramarginal gyrus, and left angular gyrus. Thirteen clinical-electrographic seizures were recorded but were poorly localized, with the most prominent activity believed to be over the left temporal head region.

The patient's seizures were not sufficiently localized to offer resective surgery. For the same reason, responsive neurostimulation and chronic subthreshold cortical stimulation were not feasible as the target location for stimulation could not be determined. The patient did not wish to proceed with a vagus nerve stimulator given concerns regarding vocal side effects as singing was very important to her. Given her multifocal epilepsy including likely extratemporal involvement, we discussed the risks and benefits of ANT and CMT DBS with the patient, and she elected to proceed with this therapy with the knowledge
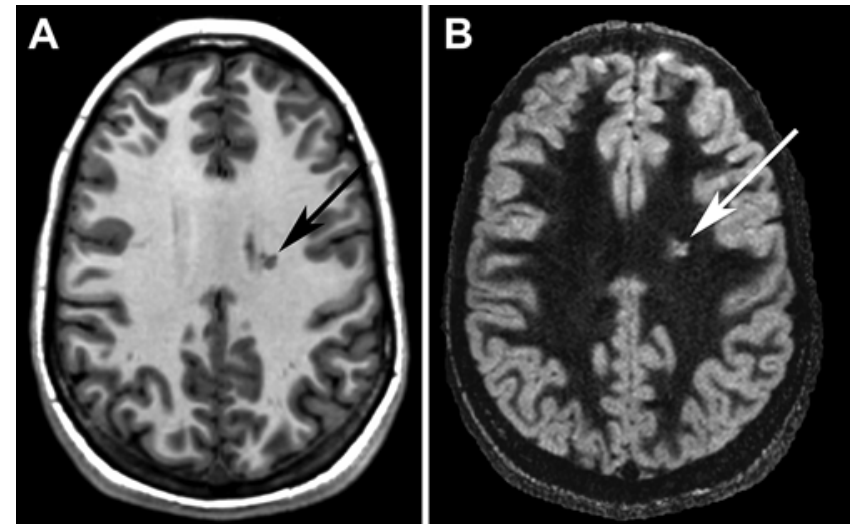

FIG. 1. Epilepsy protocol for MRI. Axial noncontrast magnetizationprepared rapid gradient echo (MP-RAGE; A) and double inversion recovery (B) images show a periventricular nodule (arrows) that followed the signal of gray matter on all sequences and was consistent with periventricular nodular heterotopia.

that the procedure was not approved by the US Food and Drug Administration.

\section{Image Acquisition}

The day prior to surgery, MRI was performed on a 3-T Siemens Prisma scanner (Siemens AG, Healthcare Sector) using a 64-channel head and neck coil. As part of the imaging protocol, noncontrast magnetization-prepared rapid gradient echo (MP-RAGE), FGATIR, and diffusion tensor imaging (DTI) sequences were obtained for targeting purposes.

Noncontrast MP-RAGE sequences were acquired with an isotropic resolution $=0.8 \times 0.8 \times 0.8 \mathrm{~mm}, \mathrm{TR}=2400$ $\mathrm{msec}, \mathrm{TE}=3.3 \mathrm{msec}, \mathrm{TI}=1050 \mathrm{msec}$, flip angle $=8^{\circ}$, parallel imaging acceleration factor (specifically, generalized autocalibrating partial parallel acquisition [GRAPPA]) = 2 , bandwidth $=220 \mathrm{~Hz} /$ pixel, turbo factor $=240$, and acquisition time $=6$ minutes 27 seconds.

The FGATIR sequences were obtained by modification of the MP-RAGE sequence, also using an isotropic resolution of $0.8 \times 0.8 \times 0.8 \mathrm{~mm}$. A nonselective inversion pulse was used with a $\mathrm{TI}=430 \mathrm{msec}$ to null white matter signal. Additional parameters included TR $=3000 \mathrm{msec}$, $\mathrm{TE}=2.2 \mathrm{msec}$, flip angle $=6^{\circ}$, slice partial Fourier $=6 / 8$, GRAPPA $=2$, bandwidth $=230 \mathrm{~Hz} /$ pixel, echo spacing $=6.8 \mathrm{msec}$, turbo factor $=180$, and acquisition time $=8$ minutes 20 seconds.

The DTI sequence consisted of a spin echo diffusionweighted echo planar imaging (EPI) sequence acquired in the axial plane utilizing simultaneous multislice (SMS) image acceleration. An isotropic resolution of $1.5 \mathrm{~mm}^{3}$ was used with TR $=3300 \mathrm{msec}, \mathrm{TE}=90 \mathrm{msec}$, echo spacing $=$ $0.94 \mathrm{msec}$, EPI factor $=140$, and bandwidth $=1232 \mathrm{~Hz} /$ pixel. A total of 64 diffusion directions with a b-value of 1500 sec/ $\mathrm{mm}^{2}$ were used, and six $b=0$ images were obtained. A slice acceleration factor of 4 was used with GRAPPA = 2 . The scan was performed twice with phase encoding in the anteroposterior and then posteroanterior directions for a total imaging time of 8 minutes 44 seconds.

Postoperatively, high-resolution, $0.8-\mathrm{mm}$, axial noncon- 
TABLE 1. Epilepsy evaluation details

\begin{tabular}{lccccccc}
\hline \multicolumn{1}{c}{ MRI Sequence } & Resolution $(\mathrm{mm})$ & $\mathrm{TR}(\mathrm{msec})$ & $\mathrm{TE}(\mathrm{msec})$ & $\mathrm{TI}(\mathrm{msec})$ & Flip Angle (degrees) & GRAPPA & Acquisition Time \\
\hline High-resolution MP-RAGE & $0.8 \times 0.8 \times 0.8$ & 2400 & 3.3 & 1050 & 8 & 2 & 6 mins 27 secs \\
\hline High-resolution FGATIR & $0.8 \times 0.8 \times 0.8$ & 3000 & 2.2 & 430 & 6 & 2 & 8 mins 20 secs \\
\hline Standard MP-RAGE & $1.2 \times 1.2 \times 1.2$ & 2300 & 2.4 & 900 & 8 & NA & 7 mins 23 secs \\
\hline
\end{tabular}

$\mathrm{NA}=$ not applicable

trast CT of the head was performed for purposes of electrode localization. In addition, a noncontrast MP-RAGE image acquired as part of the epilepsy evaluation was selected for comparison with the preoperative imaging. This scan was obtained on a 3-T GE Discovery MR750 (GE Medical Systems) and with an isotropic resolution of 1.2 $\times 1.2 \times 1.2 \mathrm{~mm}, \mathrm{TR}=2300 \mathrm{msec}, \mathrm{TE}=2.4 \mathrm{msec}, \mathrm{TI}=$ $900 \mathrm{msec}$, flip angle $=8^{\circ}$, and bandwidth $=244 \mathrm{~Hz} /$ pixel (Table 1).

\section{Operation}

After general anesthesia was induced, a Leksell frame was uniformly fit around the patient's head and secured after an appropriate preprocedural pause. Postcontrast high-resolution CT venography was then performed for stereotactic guidance. Preoperative MRI was then coregistered with premade plans to the ANT and CMT. Using the FGATIR data set, the mammillothalamic tract was directly identified in all three planes (Fig. 2). After an appropriate preprocedural pause, we started with the left ANT. We triply checked the X, Y, Z arc and collar coordinates according to plan. A burr hole was made, and we advanced the guide cannula through the dura mater down to $-15 \mathrm{~mm}$ from the target. ${ }^{33}$ We then secured the Medtronic 3389 lead to the target. Lateral fluoroscopy was used to confirm this location. To secure the lead, we used a plate composed of a standard titanium plate covered by a cutoff from the end of the plastic booties supplied in the Medtronic DBS implantation kit. We repeated the process on the right side with excellent overall results. Subsequently, we targeted both the right and left CMT/ parafascicular complex (CMTpf) by superimposing the Schaltenbrand-Wahren atlas over the patient's stereotactic MRI, and Medtronic 3387 stimulator leads were implanted with a separate burr hole for each lead. All implants were confirmed on fluoroscopy. Leads were tunneled to the left side. Leads were then connected to $60-\mathrm{cm}$ dual lead extensions (Medtronic 37082-60). Each lead was quadripolar, allowing for the insertion of two four-contact leads at the proximal portion, with the distal end consisting of an eight-contact connector. These were then tunneled and connected to a Medtronic Intellis spinal cord neurostimulator. The neurostimulator was used as it allows for stimulation through 16 contacts and connects to the quadripolar extension leads.

\section{Postoperative Course}

Two weeks after the procedure, the patient returned for initiation of stimulation and parameter optimization, which were accomplished during a four-night stay in the epilepsy monitoring unit using continuous EEG monitoring. Electroencephalography was used to monitor for subclinical and clinical seizures as well as interictal discharges, which can guide programming. ${ }^{23}$ Higher CMTpf frequencies led to facial tingling, and lower frequencies for ANT stimulation were deemed at least as effective as the higher frequencies. At the end of the patient's stay, it was concluded that the optimal individualized stimulation parameters included bilateral ANT stimulation at $7 \mathrm{~Hz}$ (pulse width [PW] $120 \mu \mathrm{sec}$ ) and bilateral CMT stimulation at $2 \mathrm{~Hz}$ (PW $120 \mu \mathrm{sec}$ ). While these parameters are not necessarily typical DBS parameters, they were chosen 1) as a result of the parameter optimization conducted during continuous scalp EEG recording for several days in the epilepsy monitoring unit, and 2) based on our prior clinical experience suggesting that lower stimulation frequencies can be helpful for these patients. For all leads, the middle two contacts received cathodal stimulation while the most proximal and distal contacts were used for anodal stimulation, yielding a type of guarded cathode elec-
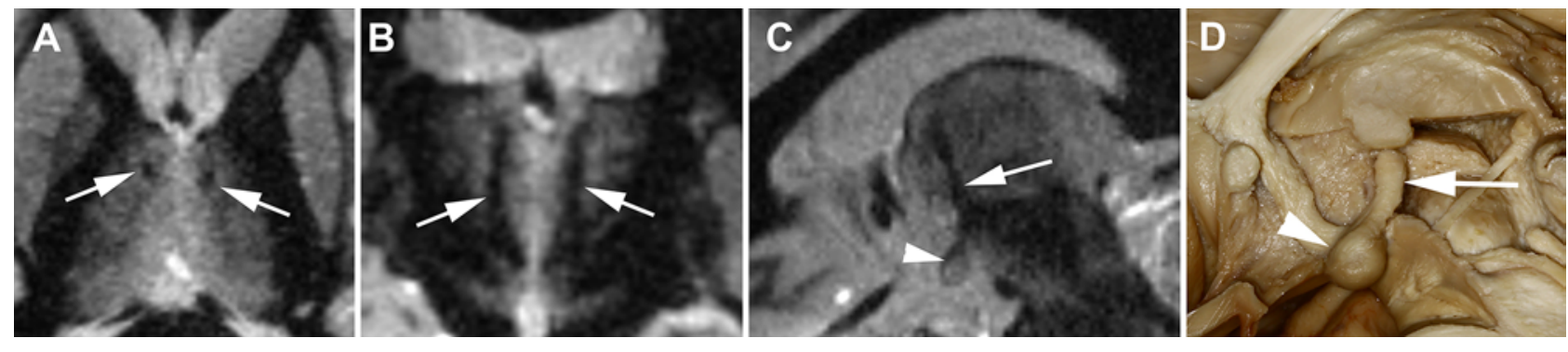

FIG. 2. Preoperative FGATIR MR images in the axial (A), coronal (B), and sagittal (C) planes. The mammillothalamic tract (arrows) is clearly visualized as a linear hypointensity extending dorsally from the mammillary body (arrowhead) to the anterior thalamus. Cadaveric dissection in the sagittal plane (D) illustrates the course of the mammillothalamic tract (arrow) originating in the mammillary body (arrowhead) and projecting to the anterior thalamus. 
trode configuration. The patient was discharged with an amplitude of approximately $3 \mathrm{~V}$ for all leads.

The DTI data underwent standard preprocessing including realignment and eddy current correction using the FMRIB Software Library (FSL; https://www.fmrib. ox.ac.uk/fsl). Distortion correction was performed utilizing the opposing phase-encoded data by means of the FSL "topup." The echo planar images were then coregistered to the structural MP-RAGE and FGATIR data using a boundary-based registration and were subsequently normalized to the MNI_ICBM_2009b_NLIN_ASYM atlas space. The orientation distribution function was estimated utilizing a high-angular resolution diffusion-weighted imaging (HARDI) method in DSI Studio (http://dsi-studio. labsolver.org/). Deterministic fiber tracking was performed from a manually defined seed region corresponding to the mammillothalamic tract on the FGATIR sequence.

To assess the final lead position and relation of the volume of tissue activated (VTA) to the mammillothalamic tract, the patient underwent postoperative head CT. The preoperative FGATIR and MP-RAGE sequences were coregistered with the postoperative CT using twostage linear registration (rigid and subsequent affine registration) using Advanced Normalization Tools. ${ }^{24}$ All volumes were subsequently normalized to the MNI ICBM_2009b_NLIN_ASYM atlas space using the SyN registration method of Advanced Normalization Tools. ${ }^{1,8}$ The Lead-DBS software package (http://www.lead-dbs. org) was used to localize DBS electrodes on the normalized postoperative CT. ${ }^{11}$ Next, the patient's final programming settings were used to model the VTA using a finite element method (FEM) solution and tissue-specific conductivity model, as implemented in Horn et al. ${ }^{12}$ To assess final electrode positioning, the center of each contact was identified in MNI space, and a 1.27-mm-diameter spatial threshold was applied to account for the lead diameter. The relationship of each contact was then assessed with the DBS Intrinsic Template Atlas (DISTAL) to isolate the ANT segment. ${ }^{6}$ The two distal-most contacts were localized within the ANT (Fig. 3). The most proximal contact was located 2.1 and $2.2 \mathrm{~mm}$ from the ANT border on the right and left, respectively. The second-most proximal contact was located 1.2 and $1.3 \mathrm{~mm}$ from the ANT border on the right and left, respectively.

The final lead positions were close to the mammillothalamic tracts bilaterally (Fig. 4). The simulated VTA showed clear overlap of the VTA with the dorsal mammillothalamic tract at its junction with the ANT. Two months following the initiation of stimulation, the patient reported 0-2 seizures per day, decreased from 5 to 7 (range 4-10) per day. Sometimes she has several days in a row without seizures, which did not happen before. Aside from some sleeping difficulties attributed to ANT stimulation, which resolved with melatonin use, she has been free of side effects. Overall, the patient has been very happy with the results, and the decrease in seizure frequency and severity has eased challenges with her ongoing college courses.

\section{Discussion}

Accurate targeting of the ANT remains challenging in

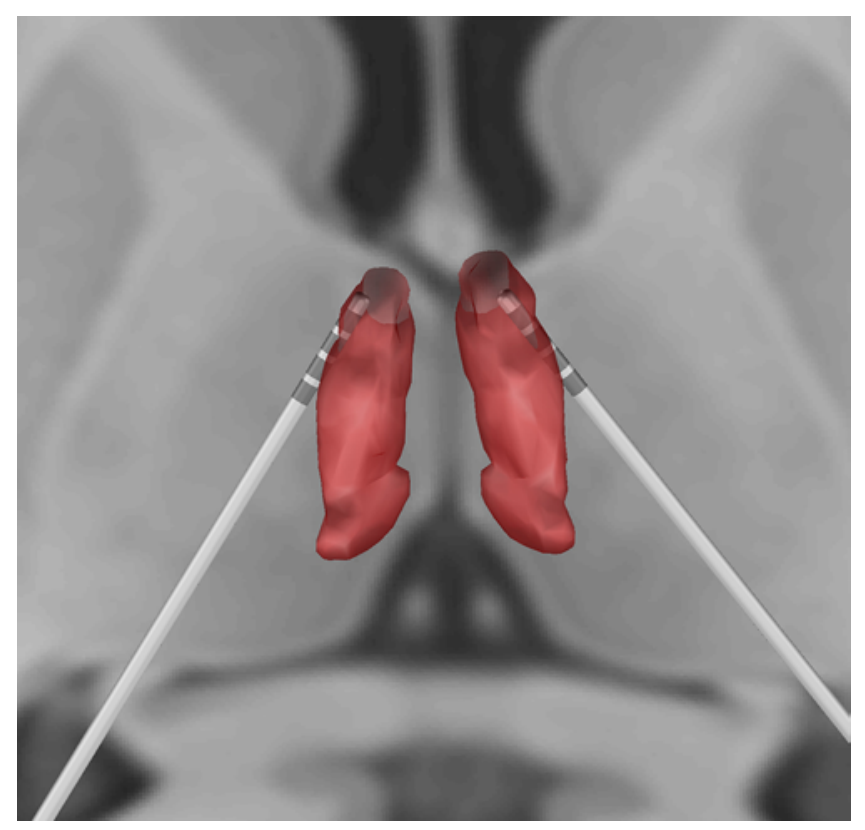

FIG. 3. Axial view showing the relationship of bilateral ANT leads with the segmented ANT (red) defined in the DBS Intrinsic Template Atlas (DISTAL). The two distal-most leads were localized within the ANT.

patients with medically intractable epilepsy. Traditional methods of targeting transventricularly compared to transfrontally have involved difficulties in hitting the target optimally, which has been addressed in the Medtronic Registry for Epilepsy (MORE) trial. ${ }^{19}$ We modified the trajectory at our institution to allow full access to the ANT; however, the target remained ambiguous because of indirect targeting. ${ }^{33}$ The two methods used for targeting include an indirect method using stereotactic coordinates and direct targeting using available imaging modalities. In the current report, we describe an improved methodology for direct targeting of the ANT using MRI with high spatial resolution, high contrast, and short acquisition time. The improvement in direct targeting minimizes individual subject variability not fully accounted for by indirect targeting methods $\mathrm{s}^{25}$ and may help to improve patient outcomes and reduce complication rates. For our case, one caveat is that the patient was implanted with electrodes targeting the bilateral CMTs as well as the ANTs, and it is unclear to what extent her benefit is related to ANT stimulation.

To date, a majority of ANT DBS studies have relied on indirect targeting methods. However, postoperative analysis reveals that there is still significant variability in electrode placement, requiring electrode revision in some cases. ${ }^{7}$ At our institution, targeting was initially performed using the coordinates described by Hodaie et al. ${ }^{10}$; we used $6 \mathrm{~mm}$ from the midline, $12 \mathrm{~mm}$ superior to the anterior commissure-posterior commissure (AC-PC), and $8 \mathrm{~mm}$ anterior to the PC. ${ }^{33}$ After this, the target was adjusted based on the superimposition of the SchaltenbrandWahren atlas in stereotactic space. As others have reported, the addition of the atlas may be of limited help, and final electrode location can be quite variable. ${ }^{30}$ Regardless 

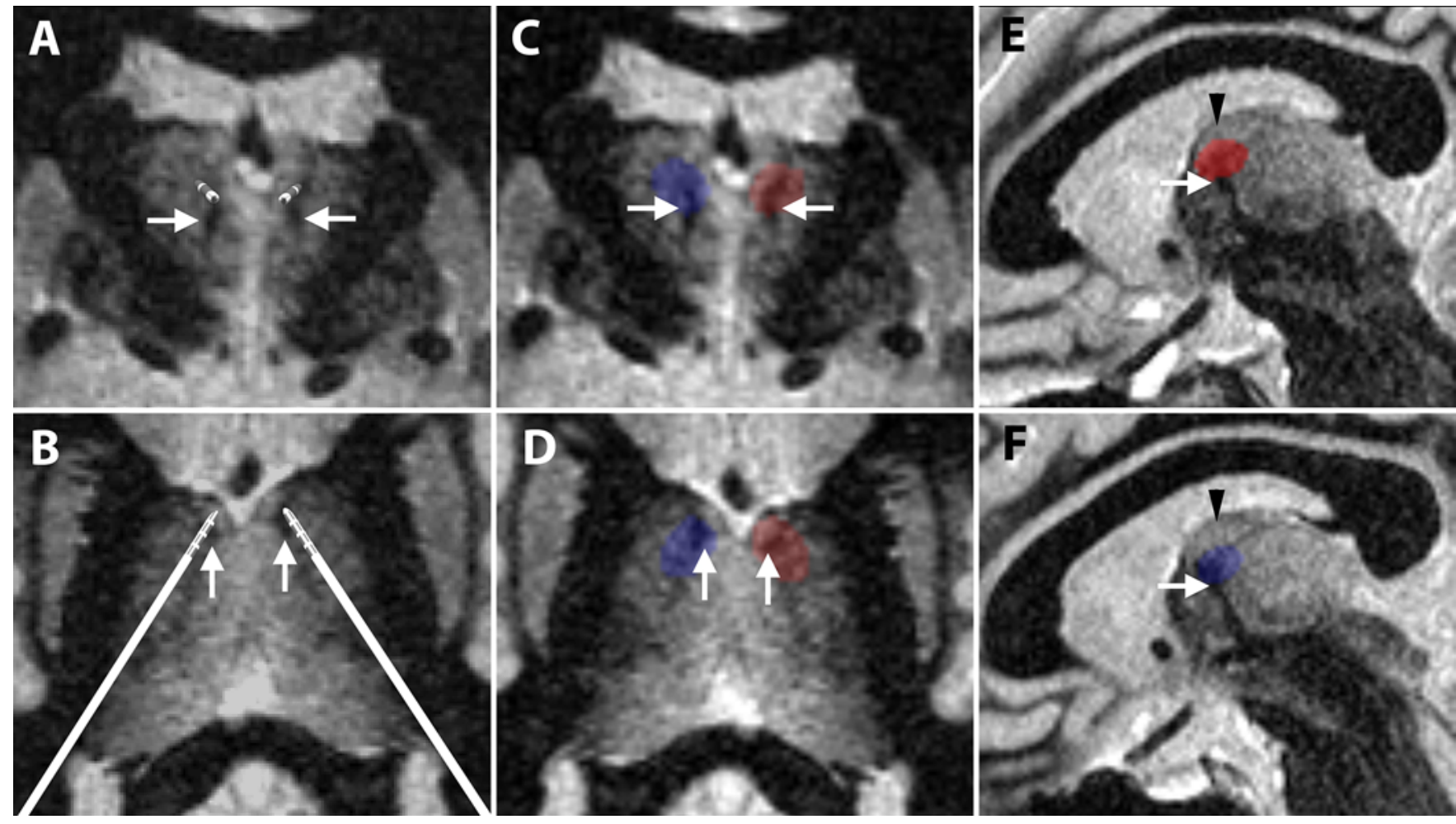

FIG. 4. The ANT DBS electrode (Medtronic 3389) localization coregistered to the preoperative FGATIR MR image. Final electrode localization is shown relative to the mammillothalamic tract (arrows) in the coronal (A) and axial (B) planes. Coronal (C), axial (D), left parasagittal $(E)$, and right parasagittal $(\mathbf{F})$ images show VTAs for the right (blue) and left (red) ANT electrodes relative to the mammillothalamic tract (arrows) and ANT (arrowheads). The VTAs are closely localized to the junction of the mammillothalamic tract and ANT on both sides.

of the indirect targeting methods utilized, the lack of accountability for variation in patient anatomy has proven problematic. For instance, an analysis of patients in the Stimulation of the Anterior Nucleus of the Thalamus for Epilepsy (SANTE) trial revealed placement outside the ANT in $10 \%$ of electrodes. ${ }^{934}$ It has become clear that improvements in direct ANT targeting are necessary to fully realize the potential benefit of ANT DBS.

Direct visualization of the ANT has been difficult because of issues in clearly visualizing the internal and external medullary lamina and the mammillothalamic tract on routine MRI sequences. Möttönen et al. previously reported the use of a short-tau inversion recovery (STIR) sequence. ${ }^{28}$ The contrast of the STIR sequence allowed some discrimination of relevant internal thalamic landmarks, such as the mammillothalamic tract, which terminates in the ANT. ${ }^{28}$ Unfortunately, due to limitations of the 2D STIR sequence, the achievable resolution in that study was $0.9 \times 0.9 \times 2 \mathrm{~mm}\left(1.62 \mathrm{~mm}^{3}\right)$ with a $0.2-\mathrm{mm}$ gap between slices. ${ }^{28}$ Thus, images were acquired in all three planes resulting in a total imaging time of more than 20 minutes $\left(7\right.$ minutes 5 seconds/scan) ${ }^{28}$ By using the FGATIR sequence, we were able to decrease the spatial resolution to $0.8 \times 0.8 \times 0.8 \mathrm{~mm}\left(0.512 \mathrm{~mm}^{3}\right)$, a nearly $70 \%$ reduction in voxel volume, while maintaining high internal contrast within the thalamus. The acquisition of isotropic resolution also allows the FGATIR images to be reformatted into any plane, which limits the needed acquisitions to one and reduces the imaging time to nearly one-third of that required for the STIR protocol.

An additional direct targeting technique utilizing the MP-RAGE sequence was proposed by Buentjen et al. ${ }^{5}$ In contrast to the method of Möttönen et al., Buentjen's technique allowed acquisition of higher-resolution 3D data sets; however, long imaging times were necessary to achieve a satisfactory contrast level within the thalamus. ${ }^{5,28}$ Buentjen et al. compared MP-RAGE sequences obtained with resolutions of 1.0 and $0.8 \mathrm{~mm}^{3}$ using multiple averaged acquisitions resulting in imaging times of 15 and 20 minutes, respectively. ${ }^{5}$ The higher-resolution $\left(0.8-\mathrm{mm}^{3}\right)$ MP-RAGE sequence was found to have inferior contrast to the $1.0-\mathrm{mm}^{3}$ sequence, leading the authors to claim that the "signal-to-noise ratio (SNR) seems to outweigh image resolution." ${ }^{\circ}$ Additionally, comparison with a shorter single-acquisition MP-RAGE sequence revealed poor internal thalamic contrast. ${ }^{5}$ Similar to Buentjen et al., we also found poor contrast and delineation of the mammillothalamic tract with higher-resolution single-acquisition MP-RAGE (Fig. 5). ${ }^{5}$ Our use of the FGATIR sequence allowed the maintenance of high contrast levels within the thalamus while still achieving the higher spatial resolution of $0.8 \mathrm{~mm}^{3}$, all while decreasing imaging time by nearly $70 \%$ (25 minutes to 8 minutes).

Diffusion tensor imaging has also been postulated as a 


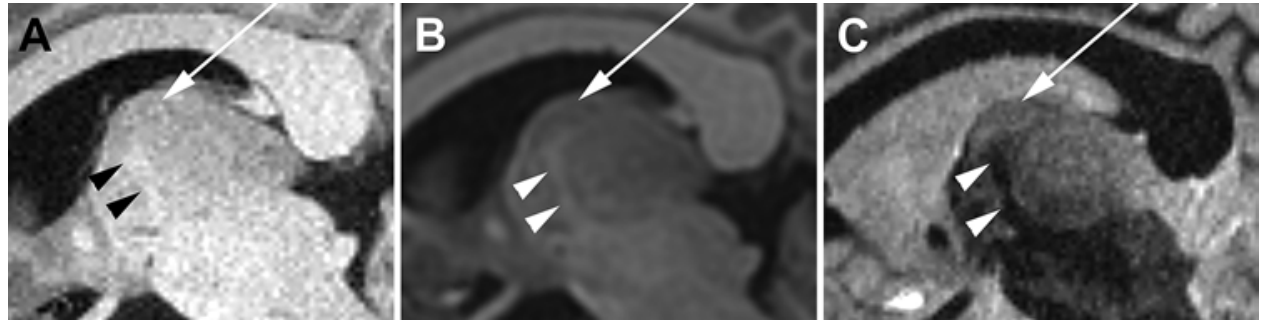

FIG. 5. Comparison of three imaging protocols in delineating the mammillothalamic tract and ANT. Sagittal MP-RAGE MR image acquired at $0.8 \mathrm{~mm}^{3}(\mathbf{A})$ shows poor delineation of the mammillothalamic tract (arrowheads) and ANT (arrow). Sagittal MP-RAGE MR image obtained at a higher resolution of $1.2 \mathrm{~mm}^{3}(\mathrm{~B})$ better illustrates the mammillothalamic tract (arrowheads) and ANT (arrow). Sagittal FGATIR MR image acquired at $0.8 \mathrm{~mm}^{3}$ (C) shows superior delineation of the mammillothalamic tract (arrowheads) within the thalamus, allowing more precise definition of the ANT (arrow).

possible means of tracking the mammillothalamic tract..$^{15}$ Kamali et al. recently reported the use of high-resolution DTI for delineating the mammillothalamic tract in healthy subjects. Using a high-performance MRI gradient system, they obtained a spatial resolution of $1 \times 1 \times$ $2 \mathrm{~mm}$ (a voxel volume nearly four times larger than our proposed FGATIR sequence). Additionally, three acquisitions were acquired per subject with an imaging time of 20 minutes. The data were then averaged across three subjects to increase the SNR, a step that is not feasible in single-subject targeting. Similar to Kamali et al., we were able to demonstrate in our current patient that the intrathalamic course of the mammillothalamic tract can be identified with a shorter acquisition scheme ( $<9$ minutes) in single subjects by using the high-performance gradients available on the Siemens Prisma scanner combined with simultaneous multislice (SMS) image acceleration (Fig. 6); however, we have not found this to be of added benefit in ANT targeting for several reasons. The long acquisition times, use of specialized equipment and MRI sequences, and added time and expertise required for fiber tracking make this technique more challenging to implement than our proposed FGATIR sequence. Additionally, inherent limitations of EPI make its use in DBS targeting potentially problematic. The spatial blurring that occurs in EPI, combined with the difficulty of achieving resolutions similar to those of 3D structural imaging acquisitions, creates more uncertainty about the precision of measures. Along these lines, the significant distortion present in EPI presents an extra challenge in accurate targeting. While DTI of the mammillothalamic tract is intriguing, we believe that it does not currently deliver any additional benefit to ANT DBS targeting compared to the high-resolution FGATIR sequence.

To augment direct targeting, centers have also tried to use microelectrode recordings to delineate boundaries of the ANT; however, this appeared to have little added value. ${ }^{28}$ In our experience, hippocampal electrodes were placed, and the Medtronic Activa PC+S system was used to record ANT stimulation-induced hippocampal evoked
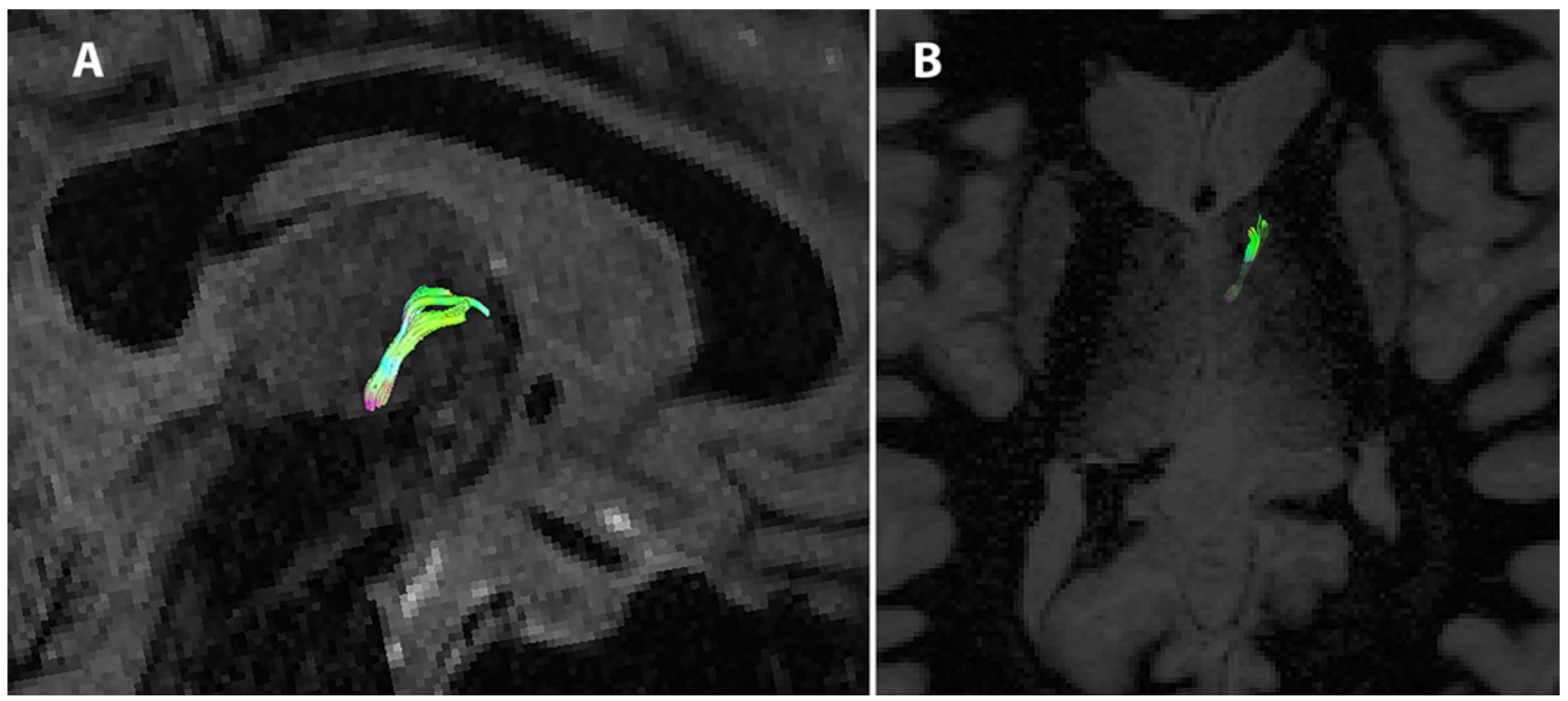

FIG. 6. Sagittal (A) and axial (B) DTI sequences in our patient coregistered to the FGATIR sequences show the course of the mammillothalamic tract within the thalamus. 
potentials as electrophysiological confirmation of appropriate placement of the ANT leads. ${ }^{33}$

An additional area of controversy regarding the placement of ANT electrodes relates to the optimal location and trajectory of leads. The two most commonly employed trajectories for placement include either a precoronal transventricular trajectory or a lateral transcortical trajectory. The precoronal transventricular trajectory requires passing two separate ependymal surfaces, which can lead to deflections and a potentially increased risk of hemorrhage. The lateral transcortical trajectory passes through eloquent cortex, such as the operculum. In addition, the lateral transcortical trajectory will provide improved mediolateral coverage, whereas the precoronal transcortical trajectory will provide improved anteroposterior coverage. The literature shows improved outcomes by targeting the superior aspect of the nucleus, with certain studies suggesting a more anterior approach targeting the anteroventral subnucleus or a more posterior approach targeting the anteromedial subnucleus..$^{18,20,34}$ While the mechanisms for the efficacy of ANT DBS have not been completely elucidated, one potential theory regarding its mechanism relies on the interruption of seizure propagation through the mammillothalamic tract as lesions and stimulation of the tract have been shown to control seizures in animal studies and clinical case reports. ${ }^{17,26,35}$ Given the variability regarding anteroposterior stimulation and the concern in crossing multiple ependymal surfaces using a transventricular approach, we employ a posterior cortical trajectory, which leads to the greatest anteroposterior coverage along with a large likelihood of crossing the mammillothalamic tract. ${ }^{33}$

While the mammillothalamic tract has the potential to be a reliable internal landmark for directly targeting the ANT, it remains difficult to visualize on routine MRI sequences. Fast gray matter acquisition T1 inversion recovery MRI has been used in DBS for movement disorders because of its improved visualization of subcortical structures as compared to standard 3-T T1- or T2-weighted imaging. ${ }^{32}$ Moreover, FGATIR is an inversion recoveryprepped gradient echo MRI pulse sequence similar to MPRAGE except that the inversion time (TI) in FGATIR is selected to null white matter signal, creating strong delineation between gray matter structures and adjacent highly myelinated white matter. The inversion time then becomes the dominant weighting factor in the contrast of this T1weighted pulse sequence in which normally dark CSF signal is bright. ${ }^{32}$ While this sequence has been used for movement disorders and for asleep targeting of subcortical structures, this report is the first on the use of FGATIR imaging to target the ANT. ${ }^{27,32}$ In using this sequence, we were able to clearly visualize the mammillothalamic tract and thus use it as a landmark to target the ANT.

\section{Conclusions}

Herein, we have described the novel use of FGATIR imaging to delineate the mammillothalamic tract and thereby target the ANT for DBS. Compared to STIR sequences or indirect methods of targeting, FGATIR appears to be a superior method of targeting; however, further prospective studies are necessary to more rigorously evaluate this imaging modality and its use in targeting the ANT.

\section{References}

1. Avants BB, Epstein CL, Grossman M, Gee JC: Symmetric diffeomorphic image registration with cross-correlation: evaluating automated labeling of elderly and neurodegenerative brain. Med Image Anal 12:26-41, 2008

2. Barron DS, Tandon N, Lancaster JL, Fox PT: Thalamic structural connectivity in medial temporal lobe epilepsy. Epilepsia 55:e50-e55, 2014

3. Bergey GK, Morrell MJ, Mizrahi EM, Goldman A, King-Stephens D, Nair D, et al: Long-term treatment with responsive brain stimulation in adults with refractory partial seizures. Neurology 84:810-817, 2015

4. Bernhardt BC, Rozen DA, Worsley KJ, Evans AC, Bernasconi $\mathrm{N}$, Bernasconi A: Thalamo-cortical network pathology in idiopathic generalized epilepsy: insights from MRI-based morphometric correlation analysis. Neuroimage 46:373-381, 2009

5. Buentjen L, Kopitzki K, Schmitt FC, Voges J, Tempelmann C, Kaufmann J, et al: Direct targeting of the thalamic anteroventral nucleus for deep brain stimulation by T1-weighted magnetic resonance imaging at 3 T. Stereotact Funct Neurosurg 92:25-30, 2014

6. Ewert S, Plettig P, Li N, Chakravarty MM, Collins DL, Herrington TM, et al: Toward defining deep brain stimulation targets in MNI space: a subcortical atlas based on multimodal MRI, histology and structural connectivity. Neuroimage 170:271-282, 2018

7. Fisher R, Salanova V, Witt T, Worth R, Henry T, Gross R, et al: Electrical stimulation of the anterior nucleus of thalamus for treatment of refractory epilepsy. Epilepsia 51:899-908, 2010

8. Fonov V, Evans AC, Botteron K, Almli CR, McKinstry RC, Collins DL: Unbiased average age-appropriate atlases for pediatric studies. Neuroimage 54:313-327, 2011

9. Gross R: Update on anterior thalamic DBS for epilepsy, presented at the International Neuromodulation Society 12th World Congress, Montreal, June 8, 2015

10. Hodaie M, Wennberg RA, Dostrovsky JO, Lozano AM: Chronic anterior thalamus stimulation for intractable epilepsy. Epilepsia 43:603-608, 2002

11. Horn A, Kühn AA: Lead-DBS: a toolbox for deep brain stimulation electrode localizations and visualizations. Neuroimage 107:127-135, 2015

12. Horn A, Reich M, Vorwerk J, Li N, Wenzel G, Fang Q, et al: Connectivity predicts deep brain stimulation outcome in Parkinson disease. Ann Neurol 82:67-78, 2017

13. Hughes EJ, Bond J, Svrckova P, Makropoulos A, Ball G, Sharp DJ, et al: Regional changes in thalamic shape and volume with increasing age. Neuroimage 63:1134-1142, 2012

14. Jiltsova E, Möttönen T, Fahlström M, Haapasalo J, Tähtinen T, Peltola J, et al: Imaging of anterior nucleus of thalamus using 1.5T MRI for deep brain stimulation targeting in refractory epilepsy. Neuromodulation 19:812-817, 2016

15. Kamali A, Zhang CC, Riascos RF, Tandon N, BonafanteMejia EE, Patel R, et al: Diffusion tensor tractography of the mammillothalamic tract in the human brain using a high spatial resolution DTI technique. Sci Rep 8:5229, 2018

16. Kerezoudis P, Grewal SS, Stead M, Lundstrom BN, Britton JW, Shin C, et al: Chronic subthreshold cortical stimulation for adult drug-resistant focal epilepsy: safety, feasibility, and technique. J Neurosurg [epub ahead of print October 20, 2017. DOI: 10.3171/2017.5.JNS163134]

17. Khan S, Wright I, Javed S, Sharples P, Jardine P, Carter M, et al: High frequency stimulation of the mamillothalamic tract for the treatment of resistant seizures associated with hypothalamic hamartoma. Epilepsia 50:1608-1611, 2009 
18. Krishna V, King NK, Sammartino F, Strauss I, Andrade DM, Wennberg RA, et al: Anterior nucleus deep brain stimulation for refractory epilepsy: insights into patterns of seizure control and efficacious target. Neurosurgery 78:802-811, 2016

19. Lehtimäki K, Coenen VA, Gonçalves Ferreira A, Boon P, Elger C, Taylor RS, et al: The surgical approach to the anterior nucleus of thalamus in patients with refractory epilepsy: experience from the international multicenter registry (MORE). Neurosurgery [epub ahead of print], 2018

20. Lehtimäki K, Möttönen T, Järventausta K, Katisko J, Tähtinen T, Haapasalo J, et al: Outcome based definition of the anterior thalamic deep brain stimulation target in refractory epilepsy. Brain Stimul 9:268-275, 2016

21. Li MCH, Cook MJ: Deep brain stimulation for drug-resistant epilepsy. Epilepsia 59:273-290, 2018

22. Lundstrom BN, Van Gompel J, Britton J, Nickels K, Wetjen $\mathrm{N}$, Worrell G, et al: Chronic subthreshold cortical stimulation to treat focal epilepsy. JAMA Neurol 73:1370-1372, 2016

23. Lundstrom BN, Worrell GA, Stead M, Van Gompel JJ: Chronic subthreshold cortical stimulation: a therapeutic and potentially restorative therapy for focal epilepsy. Expert Rev Neurother 17:661-666, 2017

24. Makris N, Goldstein JM, Kennedy D, Hodge SM, Caviness VS, Faraone SV, et al: Decreased volume of left and total anterior insular lobule in schizophrenia. Schizophr Res 83:155-171, 2006

25. Middlebrooks EH, Holanda VM, Tuna IS, Deshpande HD, Bredel M, Almeida L, et al: A method for pre-operative single-subject thalamic segmentation based on probabilistic tractography for essential tremor deep brain stimulation. Neuroradiology 60:303-309, 2018

26. Mirski MA, Ferrendelli JA: Interruption of the mammillothalamic tract prevents seizures in guinea pigs. Science 226:72-74, 1984

27. Mirzadeh Z, Chapple K, Lambert M, Evidente VG, Mahant P, Ospina MC, et al: Parkinson's disease outcomes after intraoperative CT-guided "asleep" deep brain stimulation in the globus pallidus internus. J Neurosurg 124:902-907, 2016

28. Möttönen T, Katisko J, Haapasalo J, Tähtinen T, Kiekara T, Kähärä V, et al: Defining the anterior nucleus of the thalamus (ANT) as a deep brain stimulation target in refractory epilepsy: delineation using 3 T MRI and intraoperative microelectrode recording. Neuroimage Clin 7:823-829, 2015

29. Natsume J, Bernasconi N, Andermann F, Bernasconi A: MRI volumetry of the thalamus in temporal, extratemporal, and idiopathic generalized epilepsy. Neurology 60:1296-1300, 2003

30. Osorio I, Overman J, Giftakis J, Wilkinson SB: High frequency thalamic stimulation for inoperable mesial temporal epilepsy. Epilepsia 48:1561-1571, 2007
31. Panebianco M, Rigby A, Weston J, Marson AG: Vagus nerve stimulation for partial seizures. Cochrane Database Syst $\operatorname{Rev}(4): C D 002896,2015$

32. Sudhyadhom A, Haq IU, Foote KD, Okun MS, Bova FJ: A high resolution and high contrast MRI for differentiation of subcortical structures for DBS targeting: the Fast Gray Matter Acquisition T1 Inversion Recovery (FGATIR). Neuroimage 47 (Suppl 2):T44-T52, 2009

33. Van Gompel JJ, Klassen BT, Worrell GA, Lee KH, Shin C, Zhao CZ, et al: Anterior nuclear deep brain stimulation guided by concordant hippocampal recording. Neurosurg Focus 38(6):E9, 2015

34. Wu C, D'Haese PF, Pallavaram S, Dawant BM, Konrad P, Sharan AD: Variations in thalamic anatomy affect targeting in deep brain stimulation for epilepsy. Stereotact Funct Neurosurg 94:387-396, 2016

35. Zumsteg D, Lozano AM, Wennberg RA: Mesial temporal inhibition in a patient with deep brain stimulation of the anterior thalamus for epilepsy. Epilepsia 47:1958-1962, 2006

\section{Disclosures}

Drs. Stead, Worrell, and Van Gompel report support from an NIH-funded public-private partnership grant (UH2-NS095495: Neurophysiologically Based Brain State Tracking \& Modulation in Focal Epilepsy) between Mayo Clinic and Medtronic and a Medtronic-supported investigational device exemption study (Chronically recorded deep brain nuclei/hippocampal high frequency oscillations as biomarkers of neurologic disease). The patients in this report received treatment based on compassionate, off-label use of commercially available, FDA-approved devices.

\section{Author Contributions}

Conception and design: Van Gompel, Grewal, Stead, Lundstrom, Worrell. Acquisition of data: Van Gompel, Grewal, Kaufmann, Lin, Baydin. Analysis and interpretation of data: Van Gompel, Grewal, Middlebrooks, Kaufmann, Stead, Lin. Drafting the article: Van Gompel, Grewal, Middlebrooks, Kaufmann, Lin. Critically revising the article: Van Gompel, Grewal, Middlebrooks, Kaufmann, Stead, Lundstrom, Worrell, Baydin. Reviewed submitted version of manuscript: Van Gompel, Grewal, Middlebrooks, Kaufmann, Stead, Lundstrom, Worrell, Baydin. Approved the final version of the manuscript on behalf of all authors: Van Gompel. Study supervision: Van Gompel.

\section{Correspondence}

Jamie J. Van Gompel: Mayo Clinic, Rochester, MN. vangompel. jamie@mayo.edu. 